\section{Synthetic Urines in a Buprenorphine Clinic}

David M. Embers, B.S., MS-3,

Dheepthi Arakonam Ravishankar, MBBS,

Amad Din, M.D., Roopa Sethi, M.D.

University of Kansas Medical Center,

Department of Psychiatry, Kansas City, KS

Received April 5, 2019; Accepted for publication July 24, 2019; Published online Nov. 25, 2019

\section{INTRODUCTION}

The current opioid epidemic has manifested itself in numerous ways, one of which being a dramatic rise in the way patients attempt to create a false negative urine drug screen. From urine additives to synthetic urines to prosthetics, there is no shortage of options. The use of urine analytics for drug screening purposes has been on a continuous rise since the federal government mandated the screening of all federal employees in 1988. ${ }^{1}$ As is often the case, the market to evade this screening protocol, or create a false-negative, has seen a similar growth. ${ }^{1-3}$ This case report focused on synthetic urines while on buprenorphine and outlines the numerous ways in which a patient attempted to use synthetic urine to create a false negative on her urine drug screen.

\section{CASE REPORT}

A 35-year-old female with a history of opioid use disorder, benzodiazepine dependence, amphetamine abuse, and major depressive disorder initially presented to the emergency department (ED) with opioid withdrawal symptoms after "running out" of her prescribed buprenorphine. The patient was restarted on buprenorphine while in the ED and was referred to the hospital's addiction clinic for buprenorphine maintenance where she was followed up the next day after discharge.

Upon initial interaction at her first clinic visit, she reported opiate use starting at age 23 . She stopped opiate abuse briefly during the pregnancies of her five children but would restart after delivery. She also reported intermittent amphetamine abuse for chronic fatigue and clonazepam to help with sleep and anxiety. The patient was followed by another physician at a different facility for her buprenorphine that was tapered to $2 \mathrm{mg}$ twice a day for the previous six months. She had monthly clinic visits for maintenance treatment from her previous physician. She reported being tapered to a lower dose, however, she did not reveal her amphetamine abuse leading to the taper. She reported stability and requested a month's supply of buprenorphine. We elected to provide the patient with only a week's supply of buprenorphine as we did not get the records from her previous buprenorphine provider.

Table 1 shows a series of four clinic visits where it was revealed that the patient attempted to create a false negative on her urine drug screen through the use of various urine tampering methods. Initially, she attempted to use a friend's urine who was also on buprenorphine, but she was not aware that friend was abusing opiates. On subsequent visits, she brought in synthetic urine purchased from the local gas station, attempting a different tampering method each visit. Her second visit was synthetic urine only. The third visit consisted of

\section{KANSAS JOURNAL of MEDICINE}

synthetic urine with a buprenorphine tab crushed into the urine. Her last urine was her own urine with amphetamines that she was attempting to hide.

Table 1. Patient clinic visits and results of labs.

\begin{tabular}{|l|l|l|l|l|l|}
\hline $\begin{array}{l}\text { Clinic } \\
\text { Visit }\end{array}$ & Buprenorphine & Norbuprenorphine & Ratio & Opiates & $\begin{array}{l}\text { Urine } \\
\text { Source }\end{array}$ \\
\hline Day 1 & Detected & Detected & Normal & Detected & Friend \\
\hline Day 8 & Not detected & Not detected & NA & $\begin{array}{l}\text { Not } \\
\text { detected }\end{array}$ & $\begin{array}{l}\text { Synthetic } \\
\text { urine }\end{array}$ \\
\hline Day 22 & Detected & Detected & Abnormal & Negative & $\begin{array}{l}\text { Synthetic } \\
\text { Urine with } \\
\text { Crushed } \\
\text { Buprenor- } \\
\text { phine }\end{array}$ \\
\hline Day 42 & Detected & Detected & Normal & Negative & $\begin{array}{l}\text { Patient's } \\
\text { urine } \\
\text { without } \\
\text { tampering }\end{array}$ \\
\hline
\end{tabular}

The patient revealed all of her attempted urine tampering methods after the test results were discussed with her following the Day 22 clinic visit. While she had not been using opiates, she had continued to abuse amphetamines and benzodiazepines and was attempting to hide those results. She revealed that this was the reason for her being discharged from her previous clinic and explained how she had purchased the product at a local gas station and had looked at options online. She reported that she used a product called UPass ${ }^{\mathrm{TN}}$, but said most people call it "Fetish Urine".

After revealing the tampering of urine, the patient was seen weekly and she slowly stopped use of amphetamines. Her urine drug screens revealed normal values of buprenorphine and norbuprenorphine. She was stable, participated in clinic and groups, sustained a job, and was able to support her kids.

\section{DISCUSSION}

A plethora of urine tampering options are available online ranging in price and effectiveness. These include synthetic urines, urine additives, and prosthetics. Synthetic urines claim to be an "exact match" to human urine. Some common brand names are: UPass ${ }^{\mathrm{TN}}$, Ultimate Gold, Ultra-Pure ${ }^{\circledast}$, Magnum Synthetic ${ }^{\mathrm{TM}}$, XStream ${ }^{\circledR}$, Quick Fix ${ }^{\circledast}$, Testclear, and ClearChoice ${ }^{\oplus},{ }^{1,3}$ Additionally, multiple online communities (e.g., www.urineluck.com, www.testclean.com, www.peretto.com, www.hempusa.com) have open forums that detail stories of using synthetic urine and anecdotal reports about which products did and did not work.

The online market includes urine additives, which are less prevalent, but clinicians should be aware of them. Online directions instruct to add the product to the urine sample and mix the two together. Popular brands online include Clean $\mathrm{X}^{\circledast}$, Urine AID (containing glutaraldehyde), Klear and Whizzies (containing nitrite), Urine Luck ${ }^{\circledR}$, Klear II (containing pyridinium chlorchromate, PCC), and Stealth ${ }^{\mathrm{TM}}$ (containing peroxidase and peroxide). These additives promise to "detox" the urine and "destroy unwanted substances. ${ }^{1,3}$ 


\section{KANSAS JOURNAL of MEDICINE}

SYNTHETIC URINES IN A BUPRENORPHINE CLINIC continued.

Glutaraldehyde containing substances can cause false negatives for tetrahydrocannabinol (THC) and opioids. Nitrites cause false negative on immunoassays for THC, cocaine, morphine, amphetamines, and phencyclidine and on gas chromatography (GC- MS confirmatory test) for THC. PCC can cause immunoassay false negative for THC and opiates. ${ }^{1}$

Lastly, the market includes prosthetics, which can be used in combination with synthetic urines or on their own, usually with the goal of passing a urine test where sampling collection is observed directly. Products available include The Whizzinator ${ }^{\mathrm{TM}}$, The Urinator $^{\mathrm{TM}}$, and Butt Wedge. ${ }^{1-3}$ The Whizzinator ${ }^{\mathrm{TM}}$ employs a prosthetic penis to excrete urine. The Urinator ${ }^{\mathrm{TM}}$ has a container strapped to the body with a heating unit and a tube placed near the urethra. The Butt Wedge contains a wedge-shaped container placed between users buttocks. ${ }^{1}$

While our patient was likely unaware of the specific buprenorphine to norbuprenorphine ratios needed to create a "true" false negative, physicians should be aware of it. Buprenorphine is a partial agonist at the mu opioid receptor and has poor oral bioavailability but extensive sublingual bioavailability. ${ }^{5}$ It is metabolized in the liver by N-dealkylation and gets converted to a product called norbuprenorphine. Both buprenorphine and norbuprenorphine undergo glucuronidation to inactive buprenorphine and norbuprenorphine conjugates that are excreted in the urine. ${ }^{5}$ The norbuprenorphine and buprenorphine concentration should be measured as presence of both indicates an authentic urine. The buprenorphine/norbuprenorphine ratio can be 0.2 or more. ${ }^{6}$ The ratio of buprenorphine to norbuprenorphine carries significance when attempting to identify altered urine specimens. Knowledge of these methods of urine drug screen adulteration is important for compliance versus noncompliance with treatment, diversion risk, and guiding management plans of patients.

\section{REFERENCES}

${ }^{1}$ Jaffee WB, Trucco E, Levy S, Weiss RD. Is this urine really negative? A systematic review of tampering methods in urine drug screening and testing. J Subst Abuse Treat 2007; 33(1):33-42. PMID: 17588487.

${ }^{2}$ Brock MF, Roy RC. Random urine drug testing. Anesth Analg 2009; 108(2):676. PMID: 19151314.

${ }^{3}$ Kim VJ, Okano CK, Osborne CR, Frank DM, Meana CT, Castaneto MS. Can synthetic urine replace authentic urine to "beat" workplace drug testing? Drug Test Anal 2018; 11(2):331-335. PMID: 30194711.

${ }^{4}$ Hull MJ, Bierer MF, Griggs DA, Long WH, Nixon AL, Flood JG. Urinary buprenorphine concentrations in patients treated with suboxone as determined by liquid chromatography-mass spectrometry and CEDIA immunoassay. J Anal Toxicol 2008; 32(7):516-521. PMID: 18713521.

${ }^{5}$ Elkader A, Sproule B. Buprenorphine: Clinical pharmacokinetics in the treatment of opioid dependence. Clin Pharmacokinet 2005; 44(7):661-680. PMID: 15966752.

${ }^{6}$ Böttcher M, Beck O. Evaluation of buprenorphine CEDIA assay versus GC-MS and ELISA using urine samples from patients in substitution treatment. J Anal Toxicol 2005; 29(8):769-776. PMID: 16356333. 